\title{
Status of the neutrino mass experiments KATRIN and Project 8
}

\section{Florian Fraenkle*}

Karlsruhe Institute of Technology

E-mail: florian.fraenkle@kit.edu

\begin{abstract}
A model independent, direct way to measure the neutrino masses is the investigation of the kinematics of single $\beta$-decay, via a precise measurement of the $\beta$-decay electron energy spectrum close to the endpoint. This proceedings will present the current status of two experiments intending to use this method by measuring the $\beta$-spectrum of tritium.

The KArlsruhe TRItium Neutrino (KATRIN) experiment is currently in the final phase of construction at the Karlsruhe Institute of Technology, Germany. The measurement setup consists of a high luminosity windowless gaseous molecular tritium source, a magnetic electron transport system with differential and cryogenic pumping for tritium retention, and an electro-static spectrometer section (pre-spectrometer and main spectrometer) for energy analysis, followed by a segmented detector system for counting transmitted $\beta$-electrons. Recent commissioning measurements of the main spectrometer confirmed that the spectrometer works as a high-resolution MAC-E filter and an energy resolution of about $1 \mathrm{eV}$ at $18.6 \mathrm{keV}$ is achievable.

The Project 8 experiment aims to detect coherent cyclotron radiation emitted by energetic electrons in a magnetic field in order to perform $\beta$-spectroscopy. Only recently, a dedicated test experiment was able to successfully detect synchrotron radiation emitted from a single, mildly relativistic electron for the first time, showing the feasibility of this approach and allowing for a new method to perform spectroscopy.
\end{abstract}

The European Physical Society Conference on High Energy Physics

22-29 July 2015

Vienna, Austria

* Speaker. 


\section{Introduction}

The absolute neutrino mass scale is one of the big open questions in particle physics, astrophysics and cosmology. Cosmological observations and neutrinoless double beta decay experiments provide an indirect access to the absolute neutrino mass scale, but are model-dependent. A model independent, direct approach to determine the neutrino mass is the precise investigation of weak decays such as $\beta$-decay.

In nuclear $\beta$-decay a neutron in the atomic nucleus decays into a proton, thereby emitting an electron $\left(e^{-}\right)$and an electron anti-neutrino $\left(\bar{v}_{e}\right)$. The energy released in the decay is divided between the $e^{-}$and $\bar{v}_{e}$ in a statistical way. The energy spectra of the electron is given by the well known Fermi theory of $\beta$-decay [1]:

$$
\frac{d N}{d E} \propto p\left(E+m_{e} c^{2}\right)\left(E_{0}-E\right) \sqrt{\left(E_{0}-E\right)^{2}-m_{\bar{v}_{e}}^{2} c^{4}}
$$

with the the electron energy $E$, the endpoint energy $E_{0}$, the electron mass $m_{e}$ and the average electron anti-neutrino mass $m_{\bar{v}_{e}}^{2}=\sum\left|U_{e i}\right|^{2} m\left(v_{i}\right)^{2}$. This is the incoherent sum of neutrino mass eigenstates and therefore not sensitive to phases of the neutrino mixing matrix (in contrast to neutrinoless double $\beta$-decay). As one can see in equation 1.1, it is the square of the neutrino mass $m_{v_{e}}^{2}$ that enters as a parameter. Its effect on the shape of the spectrum is significant only in a very narrow region close to $E_{0}$. The current upper limit on the neutrino mass of $2 \mathrm{eV} / \mathrm{c}^{2}$ [2] was determined from investigating the tritium $\beta$-spectrum near the endpoint of $18.6 \mathrm{keV}$ by experiments in Mainz [3] and Troitsk [4].

\section{The KATRIN experiment}

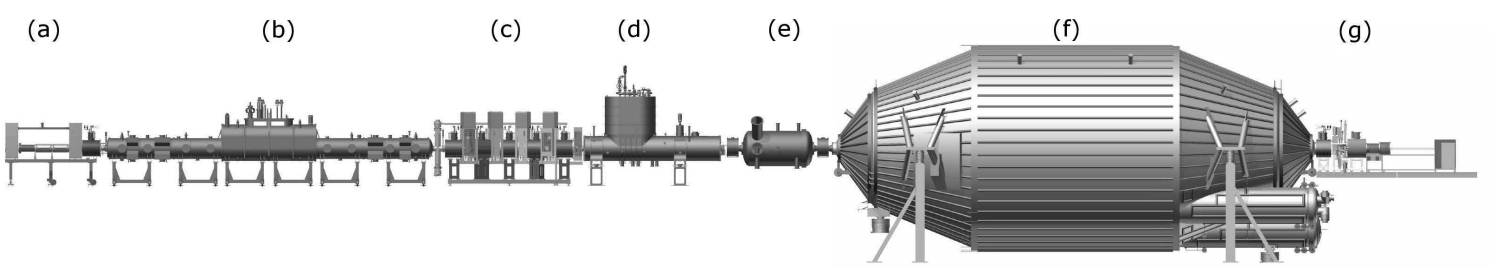

Figure 1: The KATRIN experimental setup with its main components: (a) rear section; (b) windowless gaseous tritium source (WGTS); (c) differential pumping section (DPS); (d) cryogenic pumping section (CPS); (e) pre-spectrometer; (f) main spectrometer; (g) focal plane detector.

The KArlsruhe TRItium Neutrino (KATRIN) experiment [5] is a next generation, large scale experiment to determine the mass of the electron anti-neutrino by investigating the kinematics of tritium beta decay with a sensitivity of $200 \mathrm{meV} / \mathrm{c}^{2}$. The experiment is located at the Karlsruhe Institute of Technology (KIT) in Germany. The measurement setup (see figure 1) has an overall length of $\approx 70 \mathrm{~m}$. Molecular tritium is injected into the windowless gaseous tritium source (a) where it decays with an activity of $10^{11} \mathrm{~Bq}$, thus providing a sufficient number of $\beta$-decay electrons close to the endpoint energy $E_{0}$. The tritium is removed from the beamline in the differential pumping section (b) and the cryogenic pumping section (c) while electrons from the source are magnetically guided towards the spectrometer section. Both pre-spectrometer and main spectrometer are 
operated as electrostatic retarding high pass filters of MAC-E filter type [6]. The pre-spectrometer (d) is operated as a pre-filter in order to reduce the flux of electrons into the main spectrometer (e) which performs the energy analysis of the $\beta$-decay electrons near the endpoint with an energy resolution of $\Delta E=0.93 \mathrm{eV}$ at $18.6 \mathrm{keV}$. The main spectrometer is equipped with a dual layer wire electrode system for electrostatically shielding secondary electrons from the inner vessel surface [7]. The transmitted $\beta$-decay electrons are counted in the detector system (f) with a segmented silicon detector [8].

With the arrival of the WGTS on September 10th, 2015, all major components of the experiment are now on site. Commissioning measurements of the complete beamline are expected to start in 2016, with the first tritium runs at the end of the year.
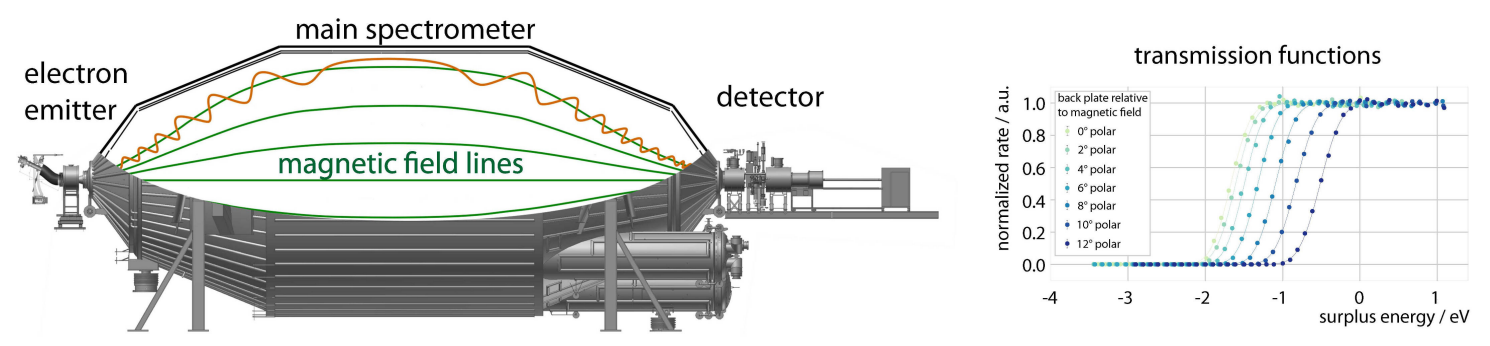

Figure 2: a) Measurement configuration during the main spectrometer and detector commissioning phase. A photoelectric electron emitter (e-gun) is mounted on the upstream side of the spectrometer, the detector system on the downstream side. b) Transmission functions for different electron polar angles at the e-gun and a spectrometer retarding potential of $-18.6 \mathrm{kV}$. The surplus energy is calculated form the difference of e-gun acceleration voltage and spectrometer retarding voltage, multiplied by the elementary charge. The onset of the transmission at negative surplus energies is due to a slightly more positive retarding potential in the analyzing plane, caused by the influence of the beam tube at ground potential on both ends of the spectrometer.

In order to investigate the performance of the main spectrometer with regard to energy resolution and background performance, a series of commissioning measurements were performed. For this purpose a photoelectric electron emitter (e-gun) was installed at the upstream side of the main spectrometer, which allows the generation of electrons of dedicated energies as well as polar angles with respect to the guiding magnetic field line. The e-gun was mounted on a manipulator in order to shoot electrons along selectable magnetic field lines through the spectrometer towards the detector system attached at the downstream side of the spectrometer (see Figure 2a)). For a fixed manipulator position and spectrometer retarding voltage, the e-gun acceleration voltage was increased in small steps and the rate at the detector was measured. This measurement was repeated for different polar angles (see figure 2b)). The width of the transmission functions is dominated by systematic effects of the e-gun, in particular energy and angular spread. The shift of the transmission functions with increasing polar angles to larger surplus energies, shows that the magnetic adiabatic collimation is working and that the KATRIN main spectrometer works as a high resolution MAC-E filter. 


\section{Project 8}

The Project 8 collaboration proposes to detect coherent cyclotron radiation emitted by energetic electrons in a magnetic field $B$ in order to perform $\beta$-spectroscopy [9]. For mildly relativistic electrons, like those in tritium decay, the relativistic shift of the cyclotron frequency $\omega(\gamma)=\frac{\omega_{0}}{\gamma}=\frac{q B}{m_{e}+E}$ can be used to determine the electron energy $E$ from the emitted radiation.

a)

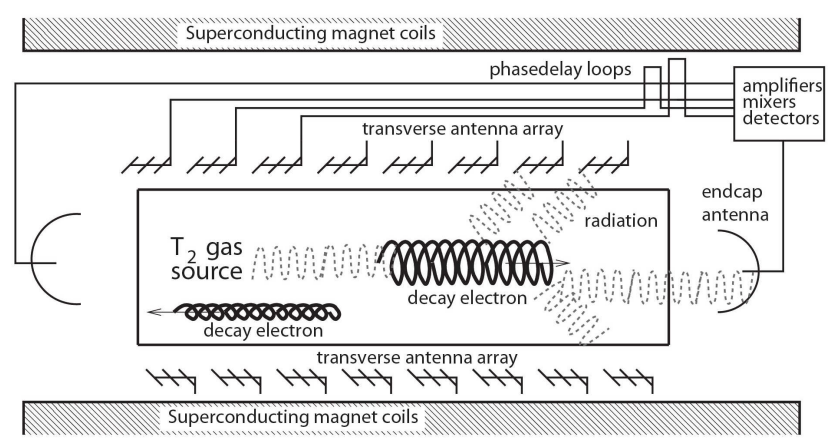

b)

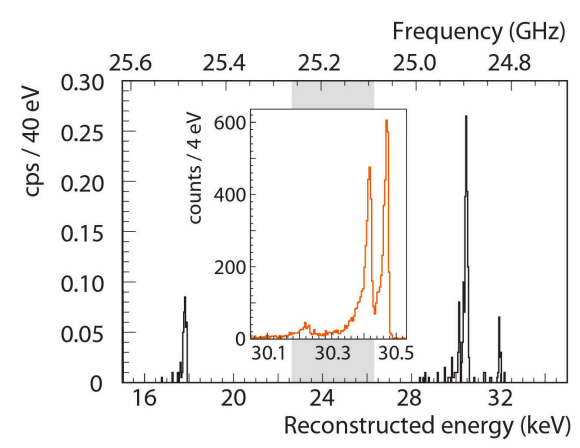

Figure 3: a) Measurement principle: electrons produced from $\beta$-decay undergo cyclotron motion in a magnetic field and emit cyclotron radiation, which is detected by an antenna array. Figure from [9]. b) Kinetic energy distribution of ${ }^{83 m} \mathrm{Kr}$ conversion electrons determined by cyclotron radiation emission spectroscopy. The spectrum shows the 17, 30 and $32 \mathrm{keV}$ complex conversion electron lines. The inset shows the region of the $30.4 \mathrm{keV}$ lines with an improved energy resolution of $15 \mathrm{eV}$ (FWHM). Figure from [10]

An experiment using a ${ }^{83 m} \mathrm{Kr}$ source, constructed by the Project 8 collaboration in order to test the feasibility of this approach, was able to detect the coherent synchrotron emission from a single electron for the first time [10]. In order to detect the cyclotron radiation, the electrons are stored in a magnetic trap. Figure 3b) shows the kinetic energy distribution of ${ }^{83 m} \mathrm{Kr}$ conversion electrons determined by their emitted cyclotron radiation. The initially achieved energy resolution of about $140 \mathrm{eV}$ (FWHM) for the $30 \mathrm{keV}$ emission lines is dominated by the spread of pitch angles of the magnetically trapped electrons. By tuning the trap to decrease the angular spread, the energy resolution could be improved to $15 \mathrm{eV}$ (FWHM) [10] at $30.4 \mathrm{keV}$ (see inset in figure 3b)).

\section{Conclusions}

Direct neutrino mass measurements are a model independent way to determine the neutrino mass. A major improvement of the neutrino mass sensitivity by one order of magnitude is expected of the KATRIN experiment, which is currently in the final phase of construction and aims to start taking neutrino mass data with tritium in 2016. The Project 8 collaboration was able to successfully detect synchrotron radiation emitted from a single, mildly relativistic electron for the first time, allowing for a new method to perform spectroscopy. This technique has the potential to go beyond the neutrino mass sensitivity reachable with electrostatic spectrometers, in the long-term.

\section{References}

[1] E. Fermi, Versuch einer Theorie der $\beta$-Strahlen, Zeitschrift für Physik 88 (1934) http://doi.org/10.1007/BF01351864 
[2] Particle Data Group, Review of Particle Physics, Chinese Physics C 38 (2014) http://doi.org/10.1088/1674-1137/38/9/090001

[3] C. Kraus et al., Final results from phase II of the Mainz neutrino mass search in tritium $\beta$ decay, The European Physical Journal C 40 (2005) http://doi.org/10.1140/epjc/s2005-02139-7

[4] V.N. Aseev et al., Upper limit on the electron antineutrino mass from the Troitsk experiment, Physical Review D 84 (2011) http://doi.org/10.1103/PhysRevD.84.112003

[5] KATRIN Collaboration, KATRIN design report, FZKA scientific report 7090 (2005) http://bibliothek.fzk.de/zb/berichte/FZKA7090.pdf

[6] G. Beamson et al., The collimating and magnifying properties of a superconducting field photoelectron spectrometer, Journal of Physics E: Scientific Instruments 13 (1980) http://doi.org/10.1088/0022-3735/13/1/018

[7] K. Valerius, The wire electrode system for the KATRIN main spectrometer, Progress in Particle and Nuclear Physics 64 (2010) http://doi.org/10.1016/j.ppnp.2009.12.032

[8] J.F. Amsbaugh et al., Focal-plane detector system for the KATRIN experiment, Nuclear Instruments and Methods in Physics Research Section A 778 (2015) http://dx.doi.org/10.1016/j.nima.2014.12.116

[9] B. Monreal and J.A. Formaggio, Relativistic cyclotron radiation detection of tritium decay electrons as a new technique for measuring the neutrino mass, Physical Review D 80 (2009) http://doi.org/10.1103/PhysRevD.80.051301

[10] D.M. Asner et al., Single-Electron Detection and Spectroscopy via Relativistic Cyclotron Radiation, Physical Review Letters 114 (2015) http://doi.org/10.1103/PhysRevLett.114.162501 